\title{
Room-temperature ultrafast carrier and spin dynamics in GaAs probed by the photoinduced magneto-optical Kerr effect
}

\author{
A. V. Kimel \\ A. F. Ioffe Physical Technical Institute, Russian Academy of Sciences, St. Petersburg 194021, Russia \\ F. Bentivegna \\ Research Institute for Materials, University of Nijmegen, Toernooiveld 1, 6525 ED Nijmegen, The Netherlands
}

V. N. Gridnev, V. V. Pavlov, and R. V. Pisarev

A. F. Ioffe Physical Technical Institute, Russian Academy of Sciences, St. Petersburg 194021, Russia

Th. Rasing

Research Institute for Materials, University of Nijmegen, Toernooiveld 1, 6525 ED Nijmegen, The Netherlands

(Received 21 December 2000; published 14 May 2001)

\begin{abstract}
The picosecond and subpicosecond dynamics of spins in intrinsic and $n$-doped GaAs is investigated at room temperature using the time-resolved, pump and probe, photoinduced, near-resonant magneto-optical Kerr effect between 1.44 and $1.63 \mathrm{eV}$. Three components with different temporal and spectral behavior are distinguished in both Kerr rotation and ellipticity, and their origin is discussed in relation with theoretical predictions and simulations. Two contributions are attributed to the splitting of the spin sublevels. The first one, present only as long as pump and probe pulses coincide in time, is a coherent response accounted for in terms of the optical Stark effect, whereas the longer decay of the second one, up to $35 \mathrm{ps,} \mathrm{is} \mathrm{attributed} \mathrm{to} \mathrm{electron} \mathrm{spin} \mathrm{relaxation.}$ The third component is assumed to arise from the difference in population of the photoexcited states and decays within a time smaller than the pulse duration, which is attributed to the energy relaxation of electrons towards the bottom of the conduction band through carrier-carrier and carrier-phonon scattering.
\end{abstract}

DOI: 10.1103/PhysRevB.63.235201

PACS number(s): 78.47.+p, 78.20.Ls, 78.40.Fy

\section{INTRODUCTION}

The ultrafast dynamics of spins in metallic ferromagnets as well as in magnetic and even nonmagnetic semiconductors ${ }^{1-6}$ has attracted intense research activity in recent years. In the case of semiconducting materials such as gallium arsenide (GaAs), essentially low-dimensional systems have been investigated, mostly at low temperature. Because of its importance for the design and fabrication of electronic devices, GaAs is a likely candidate for ultrafast, roomtemperature "spin electronics" applications, for which the spin dynamics of the carriers will be an essential parameter. The dynamics of spins can also affect the polarization noise in vertical cavity surface emitting lasers and other electronic devices functioning in the terahertz domain or at optical frequencies.

For the study presented in this paper, we used spinselective optical pumping that leads to the establishment of a transient spin polarization within the material. ${ }^{7,8}$ The use of ultrashort $(\approx 100 \mathrm{fs})$ optical pulses allows the temporal resolution of the decay of the photo-induced spin polarization in pump and probe configuration.

This decay can be monitored by measuring the linear magneto-optical effects experienced by the probe pulse as a function of the temporal delay between pump and probe pulses. Both Faraday and Kerr magneto-optical effects ${ }^{1,3,6}$ are indeed well suited to study the spin dynamics in both a broad spectral and thermal range, as well as in a thermal range. In particular, they provide a good alternative to the well-known photoluminescence techniques ${ }^{5}$ at wavelengths or temperatures for which there is hardly any measurable luminescence. For the study of thick, nontransparent samples such as those described in this paper, the Kerr effect in the reflection geometry is preferred. It must be noticed that optically induced gyrations experienced by the probe pulses depend not only on spin dynamics but also on the dynamics of the carriers themselves. Therefore, in the analysis of the photo-induced magneto-optical Kerr effect, the contributions related to carrier and spin dynamics have to be carefully separated.

We recently reported on spin polarization dynamics at room temperature in bulk cadmium telluride CdTe, ${ }^{9}$ that has the same crystallographic zinc-blende structure as GaAs, and shows very similar band diagram and band-gap energy. In this paper, we study the relaxation dynamics of spins in bulk GaAs at room temperature, both in an intrinsic and in a heavily $n$-doped sample, through time-resolved studies of the Kerr rotation and ellipticity. Special attention is also paid to the influence of carrier dynamics on the temporal behavior of the photo-induced magneto-optical Kerr effect.

\section{EXPERIMENTAL PROCEDURE}

The measurements were performed in a pump and probe configuration, using a pulsed Ti:sapphire laser with a pulse duration of approximately $100 \mathrm{fs}$ and a repetition rate of 82 $\mathrm{MHz}$, in the photon energy range of 1.44 to $1.63 \mathrm{eV}$. The setup, including a sensitive balanced detection scheme, was described in detail elsewhere. ${ }^{9}$ The intensity ratio between pump and probe pulses was set to 10:1 and they were focused on the sample to a spot diameter of about $100 \mu \mathrm{m}$ for 


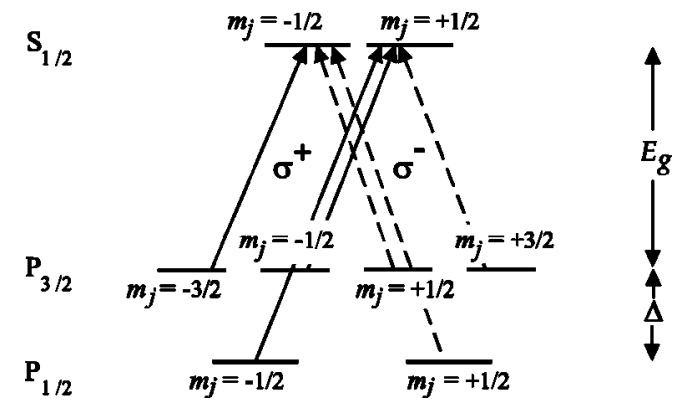

FIG. 1. Schematic electronic band structure of GaAs near the direct band gap and allowed electric dipolar transitions for lefthanded (dashed arrows) and right-handed (solid arrows) circularly polarized light. ${ }^{7}$

the pump pulse and slightly less for the probe pulse. The pump fluence on the samples was between 1 and $10 \mu \mathrm{J} \mathrm{cm}^{-2}$, producing a spatially averaged electron-hole pair density of $10^{17}$ to $10^{18} \mathrm{~cm}^{-3}$ at the band-gap resonance. The polarization of the pump beam was modulated between left and right circularly polarized states by means of a photoelastic modulator (PEM). In order to minimize coherent contributions similar to those observed in four-wave mixing experiments ${ }^{10}$ pump and probe beams were at an incidence angle of $20^{\circ}$ and $40^{\circ}$ with respect to the sample normal, respectively. The samples being thick and nontransparent, the reflection geometry was chosen. The magneto-optical Kerr rotation and ellipticity experienced by the probe beam were measured as a function of the time delay between pump and probe pulses, with a sensitivity as high as $0.2 \mu \mathrm{rad}$. The spectral profile of the laser radiation, monitored with an optical multichannel analyzer, was found to have an energy width of about $0.02 \mathrm{eV}$ over the whole photon energy range.

Two bulk GaAs samples were studied, an undoped GaAs(001) sample and a heavily $n$-doped GaAs sample with a doping concentration of $\mathrm{Si}$ atoms $n_{d}=2 \times 10^{18} \mathrm{~cm}^{-3}$. The band-gap energy $E_{g}$ of intrinsic GaAs at room temperature (Fig. 1) is approximately $1.42 \mathrm{eV}$, close to, but out of the range of the laser source.

The value of the spin split-off energy $\Delta(0.34 \mathrm{eV}$ at 300 $\mathrm{K}$ ) between $\mathrm{P}_{1 / 2}$ and $\mathrm{P}_{3 / 2}$ also makes it impossible to reach the transition between the split-off band and the conduction band around $1.74 \mathrm{eV}$. For the Si-doped sample, however, the heavy doping concentration is responsible for a nonnegligible gap narrowing $\Delta E_{g}$ that can account for an optical transition between the spin split-off band and the conduction band at an energy $E_{g}+\Delta E_{g}+\Delta$ of approximately $1.5 \mathrm{eV}$, that is, within our experimental photon energy range. An accurate estimate of $\Delta E_{g}$ is a difficult problem, since temperature corrections must also be taken into account. ${ }^{11}$ Moreover, the position of the split-off band, and thus, the value of $\Delta$ are also likely to depend on the doping concentration.

\section{EXPERIMENTAL RESULTS}

Experimentally, a linear dependence of the photo-induced magneto-optical Kerr effect on the pump fluence was found. Typical relaxation dynamics of the Kerr rotation in (a) $n$-doped GaAs and (b) undoped GaAs are shown in Fig. 2 for

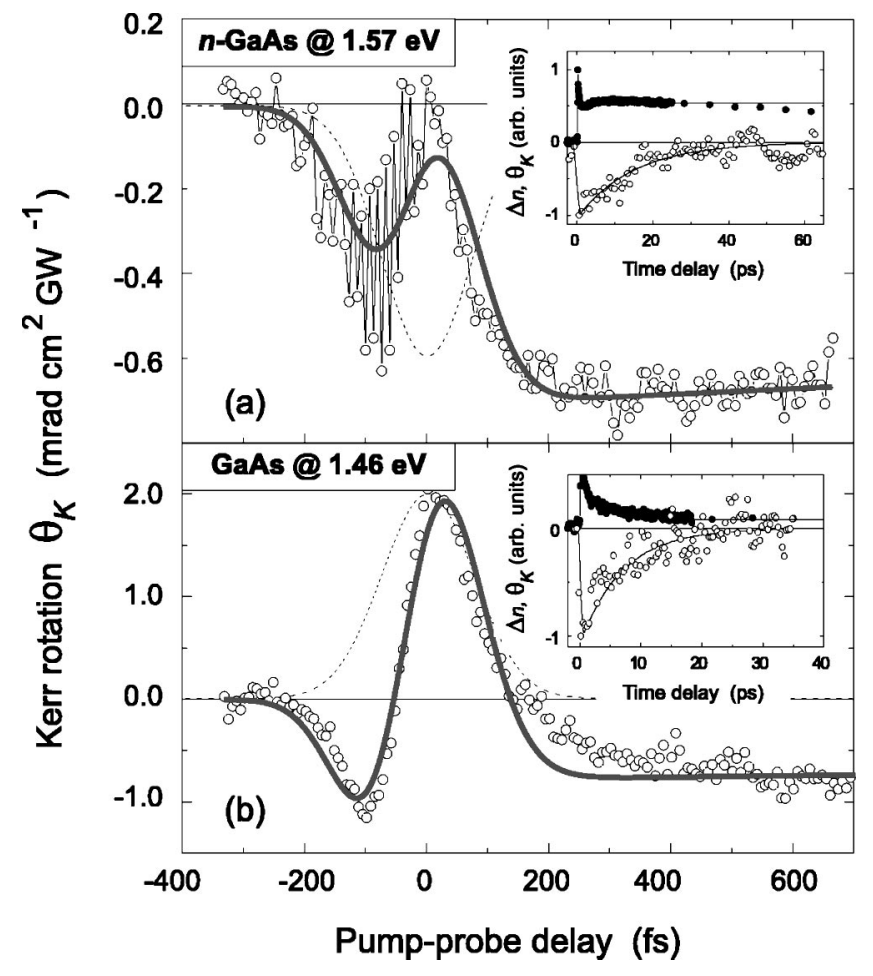

FIG. 2. Relaxation dynamics of the Kerr rotation $\theta_{K}$ (a) in $n$-GaAs and (b) in GaAs above their respective interband energy. The autocorrelation function of the pulse is indicated in arbitrary units with dashed lines. The insets show the long-term dependence of the rotation (open circles). For comparison, the insets also show the decay of the photo-induced linear birefringence $\Delta n$ due to carrier dynamics (solid circles). Solid lines are fits following Eq. (11).

optical excitations above the respective band-gap energies of the samples. In both cases, the short term behavior of $\theta_{K}$, i.e., within the first $700 \mathrm{fs}$ after excitation, is presented as well as the long-term decay of the signal (see insets). Similar relaxation dynamics were obtained for the Kerr ellipticity $\varepsilon_{K}$. The temporal shape of the autocorrelation function of the pump pulses has been included in arbitrary units for comparison (dashed lines), as deduced from the measured spectral profiles. This function is identical to the cross-correlation function of the pump and probe pulses, assuming that both pulses have the same temporal profile, and represents the period during which pump and probe pulses actually coincide in time at the sample surface.

In Fig. 2(a) fast oscillations with a period of approximately 6 fs corresponding to the experimental step of our delay line, can be observed within the duration of the crosscorrelation function of pump and probe pulses. These oscillations can be considered as experimental "artifacts" and are attributed to scattered pump light in the direction of the reflected probe pulse and subsequent interference between the two. They are actually useful in that they allow an easier determination of the zero-delay position, but they can be suppressed in order to reveal the actual magneto-optical signal onto which they are superimposed, as shown in Fig. 2(b). This can be done by introducing a periodic dephasing between pump and probe pulses and integrating the signal over sufficient time. ${ }^{12}$ 
As can be seen from Fig. 2 the initial peak of $\theta_{K}$ does not exactly follow the profile of the autocorrelation function, and shows an ultrafast relaxation contribution up to a pumpprobe delay $\Delta t$ of about $400 \mathrm{fs}$. Then a much slower relaxation component takes over, and $\theta_{K}$ totally decays within a few tens of picoseconds.

One should note that in pump and probe measurements a transient change of the refractive index of the medium can take place due to the optical Kerr effect, a third-order nonlinear optical effect, ${ }^{13}$ through the interaction of two pump photons and one probe photon. This transient relaxes with the decay of the populations of excited states and its dynamics must be carefully distinguished from the spin dynamics. In order to quantify the relaxation times involved in such refractive index transients, an additional polarizer was placed behind the PEM, resulting in a modulation of the intensity of the pump pulses, and hence, in an intentional, photo-induced change of the refractive index $\Delta n$. The pump pulses were $s$ polarized (i.e., orthogonal to the plane of incidence) whereas the polarization direction of the probe pulses was kept linear at an angle of $30^{\circ}$ relative to that of the pump pulses. The dynamics of $\Delta n$, as shown in the insets of Fig. 2 (solid circles) proves to be much slower than both components of the Kerr dynamics, confirming that the latter do indeed have a magneto-optical origin and are marginally affected by the refractive index artifacts. Moreover, the observed response allowed us to estimate that photo-induced changes of the refractive index do not exceed $1 \%$.

Within the experimental spectral range from 1.44 to 1.63 $\mathrm{eV}$, the decay time of the slow relaxation component was found between 5 and $10 \mathrm{ps}$ for intrinsic GaAs and between 15 and 35 ps for $n$-doped GaAs. The fast relaxation component showed a characteristic decay time shorter than the pulse duration for both samples. The precise determination of this parameter is limited by the pulse width.

We also observed a clear sensitivity of the short term temporal dependence on photon energy. In particular, the oscillatory behavior of $\theta_{K}$ within the first picosecond appeared only for photon energies above the interband energy, as can be seen for $n$-doped GaAs (Fig. 3). Obviously such a phenomenon results from the competition of several contributions with different spectral and temporal dependences. Thus, for a complete interpretation of the short-term behavior a theoretical derivation of these various contributions must be carried out.

\section{THEORETICAL ANALYSIS OF THE EXPERIMENTAL DATA}

As is well known, the magneto-optical Kerr effect is a linear function of the magnetization inside a medium. ${ }^{14}$ This magnetization can be caused by an external magnetic field, an intrinsic magnetic ordering or, as described in this paper, a process such as optical orientation due to photoexcitation. The latter can be phenomenologically described as the inverse Faraday effect, ${ }^{15}$ in which circularly polarized light induces in a medium an electric displacement vector $\mathbf{D}$ that, in turn, creates a local magnetization $M_{i}=\chi_{i j k}\left[D_{j} D_{k}^{*}\right]$. However, this approach to photo-induced magneto-optical

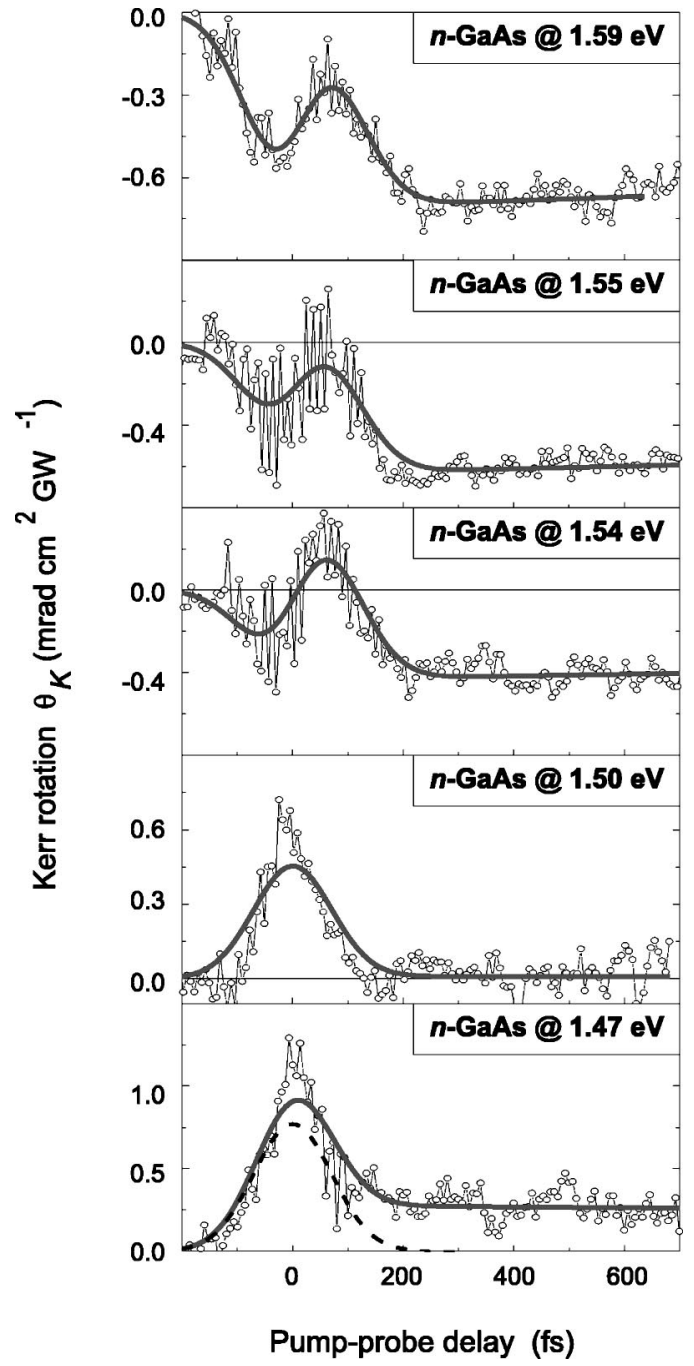

FIG. 3. Short-term dynamics of the Kerr rotation $\theta_{K}$ in $n$-GaAs for different photon energies below and above the band-gap energy. Solid lines are fits following Eq. (11).

effects as the result of an optically induced magnetization does not completely describe the observed phenomena. ${ }^{6} \mathrm{~A}$ more detailed description of the photo-induced magnetooptical Kerr effect is therefore needed.

A complex magneto-optical Kerr effect $\Theta_{K}$ arises whenever there is a difference between the optical coefficients of a medium for right-handed and left-handed circularly polarized light:

$$
\Theta_{K}=\varepsilon_{K}+i \theta_{K}=-\frac{1}{n\left(n^{2}-1\right)}\left(\chi^{(+)}-\chi^{(-)}\right),
$$

where $\chi^{(+)}$and $\chi^{(-)}$are the dielectric susceptibilities of the material for right-handed and left-handed circularly polarized light, respectively. In the particular case of optical pumping with a circularly polarized light pulse of a medium with a band structure such as that of GaAs (Fig. 1) bleaching can occur, where one of two possible transitions is selectively excited over the other (e.g., from $m_{j}=-\frac{3}{2}$ to $m_{j}=$ $-\frac{1}{2}$ or from $m_{j}=\frac{3}{2}$ to $m_{j}=\frac{1}{2}$, depending on the helicity of 
the light polarization). During and after the excitation, the transition probability for probe photons of the same energy will be smaller for this already partially pumped transition. As a result, the optical coefficients of the medium probed with circularly polarized pulses of opposite helicities are different, due to the difference of populations of the ground and excited sublevels. Consequently a contribution, that can be named population induced, appears in $\Theta_{K}$. This contribution was studied in $\mathrm{Cd}_{0.75} \mathrm{Mn}_{0.25} \mathrm{Te}\left(E_{g}=1.95 \mathrm{eV}\right)$ at $\hbar \omega$ $=1.17 \mathrm{eV}$, and the relevant effect was found to be quadratic in the intensity of light. ${ }^{16}$

On the other hand, whenever there is a spectral dispersion of the optical properties of a medium, the energy splitting of the spin sublevels also leads to magneto-optical effects. In the case of the 'ordinary' magneto-optical Faraday and Kerr effects the splitting is caused by an external magnetic field or by a spontaneous magnetization via the Zeeman effect. In contrast, a photo-induced lifting of the magnetic quantum number degeneracy can be caused by the optical Stark effect ${ }^{17}$ or by many-body effects. ${ }^{18}$ Consequently, another, splitting-induced, contribution to the photo-induced magneto-optical Kerr effect, can arise.

In order to account for the photo-induced origin of the complex Kerr rotation, as well as the temporal shape of the pulse, Eq. (1) can be rewritten as:

$$
\Theta_{K}=-\frac{1}{n\left(n^{2}-1\right) \int_{-\infty}^{\infty} d t\left|E_{0}\right|^{2}} \int_{-\infty}^{\infty} d t\left(P^{(+)}-P^{(-)}\right) E_{0}^{*},
$$

where $E_{0}$ is the electric field of the right-handed as well as left-handed circularly polarized probe pulse and $P^{( \pm)}$is the third-order polarization induced in the material and depends quadratically on the pump electric field $E$ and linearly on the probe field $E_{0} \cdot{ }^{19}$ Contrary to $P^{( \pm)}$, the refractive index $n$ of the medium can in first approximation be considered as practically unchanged by the pump pulse, since photo-induced changes of $n$ do not exceed $1 \%$, as shown above. It should be emphasized that Eq. (2) describes any change of the probe polarization state, irrespective of its microscopic origin.

The photo-induced macroscopic polarization $P^{( \pm)}$is related to the wave-vector-dependent interband optical polarizations $P_{s j}(\mathbf{k})$, i.e., to the interband elements of the density matrix, for transitions between states in the conduction subband with the spins $s= \pm \frac{1}{2}$ and states in the valence band with projections of the total angular momenta $j= \pm \frac{3}{2}, \pm \frac{1}{2}$, via:

$$
P^{( \pm)}(t)=\sum_{\mathbf{k}, s, j}\left|\psi_{k}(0)\right|^{2} d_{s j}^{ \pm} P_{s j}(t, \mathbf{k}),
$$

where the $d_{s j}^{ \pm}=\left(d_{x}\right)_{s j} \pm i\left(d_{y}\right)_{s j}$ are the dipolar matrix elements and $|\psi(0)|^{2}$ is the Sommerfeld enhancement factor. The interband polarizations $P_{s j}(\mathbf{k})$ are calculated, together with the electron density matrix elements $n_{s s^{\prime}}$, from the semiconductor Bloch equations (SBE) (Ref. 20) (taking $\hbar$ $=1)$ :

$$
\begin{aligned}
& i \frac{d P_{s j}(\mathbf{k})}{d t}=\left[\epsilon_{s j}(\mathbf{k})-\frac{i}{T_{2}}\right] P_{s j}(\mathbf{k})-\Omega_{s j}(\mathbf{k})\left(1-n_{s s}-n_{j j}\right), \\
& \frac{d n_{s s^{\prime}}(\mathbf{k})}{d t}= i \sum_{s}\left[\Omega_{s j}(\mathbf{k}) P_{s^{\prime} j^{\prime}}^{*}(\mathbf{k})-\Omega_{s^{\prime} j}^{*}(\mathbf{k}) P_{s j}(\mathbf{k})\right] \\
&-\sum_{s_{1} s_{2}} \Gamma_{s s^{\prime} s_{1} s_{2}} n_{s_{1} s_{2}}(\mathbf{k}),
\end{aligned}
$$

and an equation similar to Eq. (5) for the hole density-matrix elements $n_{j j^{\prime}}(\mathbf{k})$. Coefficients $\Gamma_{s s^{\prime} s_{1} s_{2}}$ determine the relaxation rates of the intraband elements $n_{s s^{\prime}}$ of the density matrix and include charge and spin relaxation terms.

For simplicity, we neglect the effect of the valence-band mixing. In Eq. (4) $\epsilon_{s j}(\mathbf{k})=k^{2} / 2 \mu_{s j}+E_{g}+\Sigma_{s}+\Sigma_{j}$, where $\mu$ is the reduced mass, $E_{g}$ is the band-gap energy, and $\Sigma_{s}(\mathbf{k})$ and $\Sigma_{j}(\mathbf{k})$ are self energies. $T_{2}$ is the interband dephasing time and the renormalized Rabi energy $\Omega_{s j}$ is given by:

$$
\Omega_{s j}(\mathbf{k})=\mathbf{d}_{s j} \cdot \mathbf{E}+\sum_{\mathbf{q}} V(q) P_{s j}(\mathbf{k}+\mathbf{q}),
$$

where $\mathbf{E}$ is the sum of the pump and probe electric fields and $V(\mathbf{q})$ is the Coulomb potential.

Interband dipolar moments $\mathbf{d}_{s j}$ determine the selection rules. For transitions from the heavy-hole subband $\mathbf{d} \frac{3}{2}, \frac{1}{2}=$ $-d \mathbf{e}_{-}$and $\mathbf{d}_{-\frac{3}{2},-\frac{1}{2}}=-d \mathbf{e}_{+}$, where $d$ is the magnitude of the dipolar moment and $\mathbf{e}_{ \pm}=\mathbf{e}_{x} \pm i \mathbf{e}_{y}, \mathbf{e}_{x}$ and $\mathbf{e}_{y}$ being the Cartesian unit vectors.

After solving the SBE numerically and calculating the macroscopic polarization $P(t)$, one can finally determine the Kerr rotation $\theta_{K}$ and ellipticity $\varepsilon_{K}$ by using Eq. (2).

In order to complete this analysis and to account for the two types of contributions to the dynamics distinguished above, we need to discuss briefly the sources of nonlinearity in SBE, which can be considered threefold:

(i) A phase-space filling term, arising from the Pauli exclusion principle. ${ }^{21}$ As mentioned above, when the semiconductor is pumped with a circularly polarized light pulse, the transition selectively excited over the other responds to the following probe pulse with a somewhat weaker oscillator strength and a population-induced contribution appears. The phase-space filling contribution brings information about the time-dependent redistribution of carriers due to carriercarrier and carrier-LO phonon interactions. ${ }^{22,23}$

(ii) A term accounting for the energy renormalization or self-energy terms, which describe the changes in one-particle dispersion relations due to the Coulomb interaction. In the simplest, mean-field approximation, the self-energies $\Sigma_{s}(\mathbf{k})$ and $\Sigma_{j}(\mathbf{k})$ are given by: ${ }^{17}$

$$
\Sigma_{s}(\mathbf{k})+\Sigma_{j}(\mathbf{k})=-\sum_{\mathbf{q}} V(\mathbf{q})\left[n_{s s}(\mathbf{k}+\mathbf{q})+n_{j j}(\mathbf{k}+\mathbf{q})\right],
$$

and arise from the reduction of the repulsive interaction among carriers with equal spin due to the Pauli exclusion principle. After the semiconductor has been optically excited 


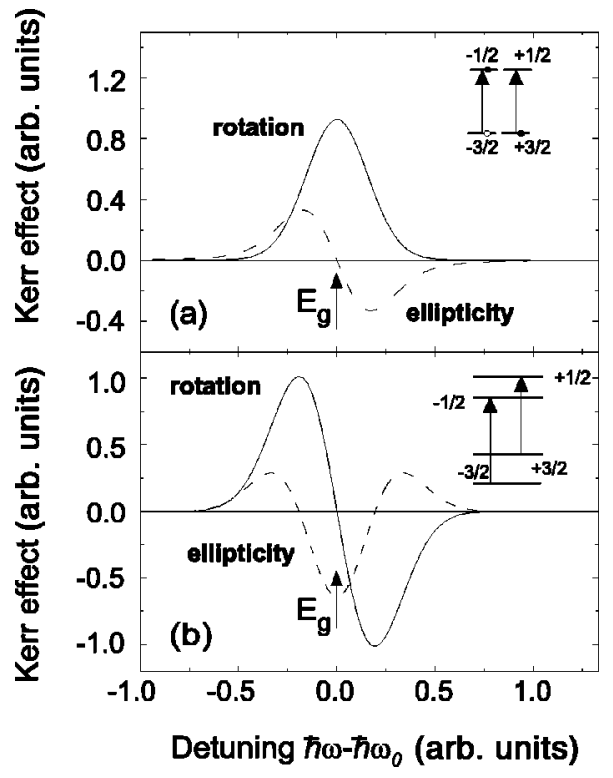

FIG. 4. Schematic optical transition configuration and simulated spectra of (a) the population-related, photo-induced magnetooptical Kerr effect and (b) the energy splitting-related magnetooptical Kerr effect around a resonance at $\hbar \omega_{o}$.

with circularly polarized pump light, the spin system is no longer in equilibrium, i.e., $n \frac{1}{2}, \frac{1}{2} \neq n_{-} \frac{1}{2},-\frac{1}{2}$, and a spin splitting of the energy bands arises, before decreasing again during the process of spin relaxation.

(iii) Finally, a third source of optical nonlinearity, related to the renormalization of the optical electric field due to the Coulomb interaction (local-field effects) and accounted for in the renormalized Rabi energy $\Omega_{s j}$. Local-field effects are essential only when the optical dephasing time $T_{2}$ is greater than the pulse duration.

A quantitative simulation of the optical properties of photo-excited semiconductors with full consideration of microscopic interactions, spin-dependent effects of band-gap renormalization, taking into account the transitions from both heavy-hole and light-hole bands is a self-consistent problem ${ }^{23}$ beyond the scope of this paper. However, a qualitative simulation of the spectral behavior of the populationinduced and splitting-induced contributions to the photoinduced magneto-optical Kerr effect can be achieved by solving the optical Bloch equations separately for phasespace filling (i) and spin-dependent band-gap renormalization effects (ii) within the approximation of an ensemble of two-level systems, which in principle only applies to excitonic transitions. ${ }^{20}$ The results of such a simulation are presented in Fig. 4.

Figure 4 clearly shows the different spectral dependences of the two types of contributions to the photo-induced magneto-optical Kerr effect. Indeed, the population-related component shows an extremum of the Kerr rotation around the electronic transition, accompanied by a sign reversal of the Kerr ellipticity at the same point, in agreement with the Kramers-Kronig relations. This spectral behavior is sometimes called "paramagnetic." ",14 In contrast, the splittinginduced component shows an opposite spectral dependence, called "diamagnetic," i.e., a sign reversal of the Kerr rotation and an extremum of the Kerr ellipticity at the resonance frequency.

In order to compare our experimental results with a phenomenological theory, we can derive a simple fitting relation for $\Theta_{K}$ from the following considerations.

The complex Kerr rotation can be related to the photoinduced electron and hole densities as follows: ${ }^{24}$

$$
\begin{aligned}
\Theta_{K} \propto \sum_{s, j} \sigma \int_{-\infty}^{\infty} d t\left\{E_{\sigma}(t)\left|d_{j s}\right|^{2}|\psi(0)|^{2}\right. \\
\left.\quad \times \int_{-\infty}^{t} d t^{\prime} E_{\sigma}^{*}\left(t^{\prime}\right)\left(e^{i \epsilon_{s j}\left(t-t^{\prime}\right)}\left[n_{s s}\left(t^{\prime}\right)+n_{j j}\left(t^{\prime}\right)\right]\right)\right\},
\end{aligned}
$$

where $\sigma=s-j$ denotes the angular momentum of the photons engaged in the transitions.

Providing both the pump and the probe pulses are Gaussian, the optical pump intensity takes the following form:

$$
I(t) \propto \frac{1}{\sqrt{2 \pi} w} \exp \left(-\frac{t^{2}}{2 w^{2}}\right),
$$

where $w$ is the rms width of the pulse.

For interband excitations in GaAs the dephasing time $T_{2}$ is much shorter than the experimental pulse duration. ${ }^{25}$ Hence, we can approximately represent the photo-induced carrier density as:

$$
n_{s s}(t)+n_{j j}(t)=a e^{-\left(t^{2} / 2 w^{2}\right)}+b e^{-\left(t / \tau_{1}\right)}+c e^{-\left(t / \tau_{2}\right)} .
$$

Substituting this expression into Eq. (8) and performing the integration we obtain for the magneto-optical Kerr rotation:

$$
\begin{aligned}
\theta_{K}= & \theta_{K}^{i n s t}+\theta_{K}^{t d 1}+\theta_{K}^{t d 2} \\
= & A \exp \left(-\frac{\tau_{d}^{2}}{4 w^{2}}\right) \\
& +\frac{B}{2} \exp \left(\frac{w^{2}}{\tau_{1}^{2}}-\frac{\tau_{d}}{\tau_{1}}\right)\left[1-\operatorname{erf}\left(\frac{w}{\tau_{1}}-\frac{\tau_{d}}{2 w}\right)\right] \\
& +\frac{C}{2} \exp \left(\frac{w^{2}}{\tau_{2}^{2}}-\frac{\tau_{d}}{\tau_{2}}\right)\left[1-\operatorname{erf}\left(\frac{w}{\tau_{2}}-\frac{\tau_{d}}{2 w}\right)\right],
\end{aligned}
$$

where $\tau_{d}$ is the delay time between pump and probe pulses. The first term in this expression describes the instantaneous, coherent response and the other two describe relaxing responses with relaxation times $\tau_{1}$ and $\tau_{2}$, respectively. We can assume that the decay times $\tau_{1}$ and $\tau_{2}$ describe the relaxation of carrier densities and spins, respectively. However, because of its approximate character, Eq. (11) does not describe $\theta_{K}\left(\tau_{d}\right)$ properly at small $\tau_{d}$. For this reason, the relaxation time $\tau_{1}$ should rather be considered as an effective parameter. Its physical interpretation depends on the details of the excited state of the semiconductor and can change with the central frequency of the exciting radiation. 
A relation similar to Eq. (11) stands for the Kerr ellipticity $\varepsilon_{K}$, with amplitudes respectively denoted $A^{\prime}, B^{\prime}$, and $C^{\prime}$. For the fitting procedure of the experimental Kerr rotation dynamics the respective amplitudes $A, B$, and $C$ of the three contributions were set as the sole fitting parameters, while $w, \tau_{1}$, and $\tau_{2}$ were fixed. Providing $\tau_{2} \gg w$, the value of $\tau_{2}$ does not influence the short-term temporal behavior (see Eq. 11). We also found that the best fit of the data is obtained for $\tau_{1}$ around $20 \mathrm{fs}$ in $n$-doped GaAs and $30 \mathrm{fs}$ in intrinsic GaAs.

The spectral dependence of the amplitudes $A, B, C$, and $A^{\prime}, B^{\prime}, C^{\prime}$, as deduced from the fitting carried out with $w$ $=50 \mathrm{fs}, \tau_{1}=20 \mathrm{fs}$ and $\tau_{2}=10 \mathrm{ps}$, are plotted for both $\theta_{K}$ and $\varepsilon_{K}$ in Figs. 5(a), 5(b), and 5(c), respectively, for $n$-doped GaAs. We should note that the fitting procedure of the Kerr ellipticity dynamics could not be performed with three independent fitting parameters. Taking into account the similarity of the spectral dependences of $A$ and $C$, the simplifying condition $A^{\prime}=C^{\prime}$ was assumed for Kerr ellipticity.

The spectra are similar to those observed in CdTe. ${ }^{9}$ For $n$-doped GaAs, the spectral dependences of $A$ and $C$ are also similar, as are indeed the spectra of $A^{\prime}$ and $C^{\prime}$, showing a sign reversal of the Kerr rotation around $1.50 \mathrm{eV}$ and a corresponding extremum of the Kerr ellipticity near the same energy. In strong contrast to this behavior, the spectral dependences of $B$ and $B^{\prime}$ are characterized by an extremum of the rotation and a corresponding sign reversal of the ellipticity around the larger photon energy of $1.56 \mathrm{eV}$.

The insets of Fig. 5 show the spectral dependences of the amplitudes $A, B, C$, and $A^{\prime}, B^{\prime}, C^{\prime}$ deduced from the fits of the Kerr effect dynamics in undoped GaAs. The fitting procedure was carried out with $w=50 \mathrm{fs}, \tau_{1}=30 \mathrm{fs}$ and $\tau_{2}$ $=10$ ps. For this sample, the direct interband gap energy $(1.42 \mathrm{eV})$ is out of reach of the Ti:sapphire laser used for the experiments. However, the spectra obtained for $A$ and $C$, as well as for $B$, are indeed similar to those observed in $n$-doped GaAs when translated towards lower photon energies.

\section{DISCUSSION}

From the comparison of the spectra obtained for $n$-doped GaAs and the simulated spectra of the various contributions to the photo-induced magneto-optical Kerr effect, one observes a qualitative agreement between the calculated splitting-induced spectrum [Fig. 4(b)] and the spectral behavior of amplitudes $A$ and $C$ [Figs. 5(a) and 5(c)], where the rotation changes sign and the ellipticity reaches a maximum at $1.50 \mathrm{eV}$. This value can be assigned to the electron transition energy between the top of the split-off valence band and the bottom of the conduction band (Fig. 1) in heavily $n$-doped GaAs. A further qualitative agreement can also be found between the spectral dependence of the calculated population-induced contribution [Fig. 4(a)] and that of the amplitude $B$ [Fig. 5(b)], with a maximum of the Kerr rotation and a sign change of the ellipticity at $1.56 \mathrm{eV}$. The discrepancy between the transition energy determined from the spectral dependence of $A$ (and $C$ ) and that of $B$ can be attributed to the rough assumption made for the fitting of ellipticity data.

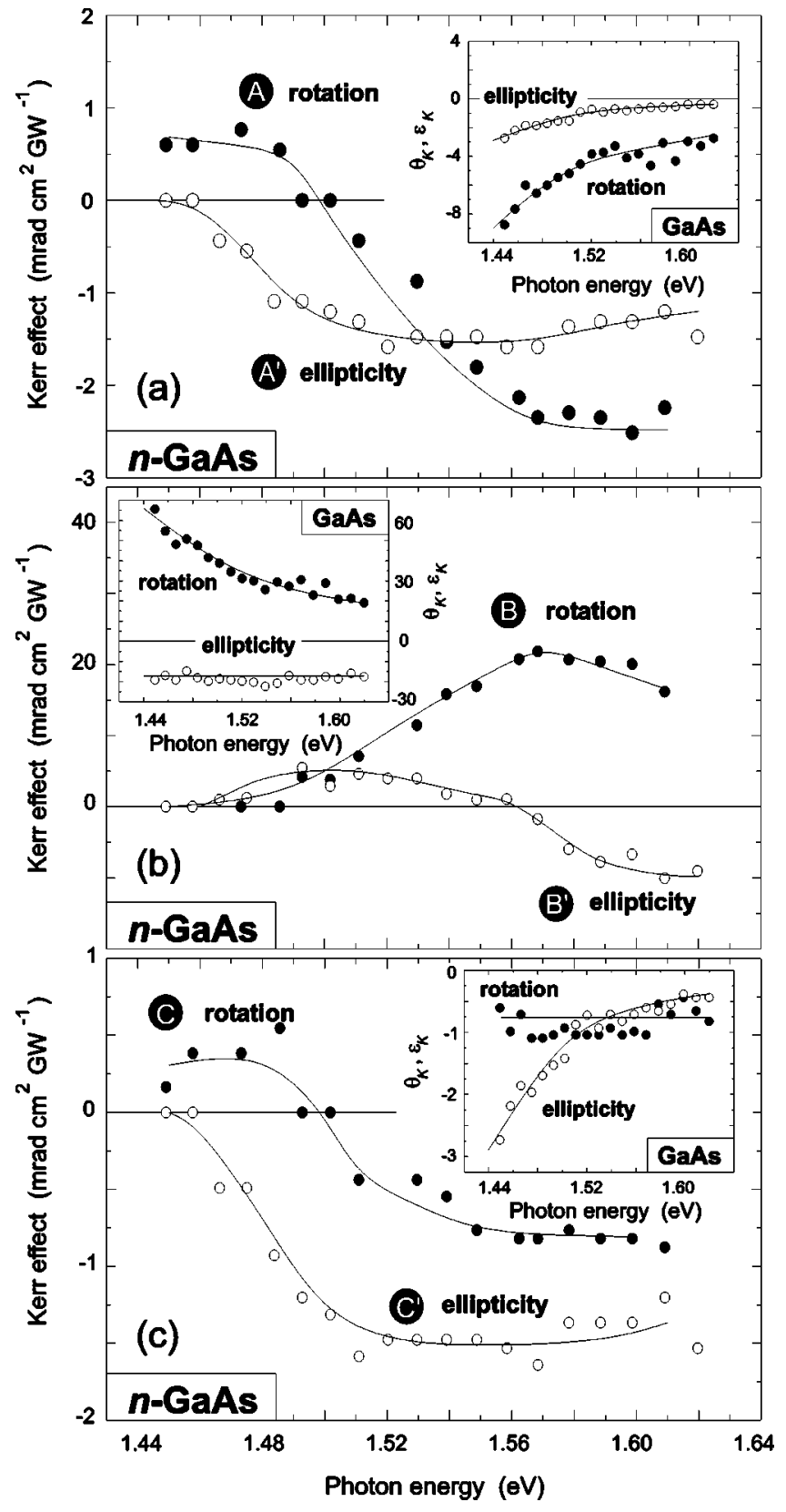

FIG. 5. Spectral dependence of the amplitudes of the three contributions to the Kerr rotation $\theta_{K}$ and ellipticity $\varepsilon_{K}$ in $n$-doped GaAs and undoped GaAs (inset), namely (a) the amplitudes $A$ and $A^{\prime}$ of the instantaneous contribution, (b) the amplitudes $B$ and $B^{\prime}$ of the subpicosecond relaxing component, and (c) the amplitudes $C$ and $C^{\prime}$ of the slowly relaxing component. Lines are guides to the eye.

The same observations can be made for intrinsic GaAs, where the spectra, as noted above, are similar to those observed in $n$-doped GaAs, providing a translation towards lower photon energies is made. All these features allow us to conclude that in both $n$-doped GaAs and intrinsic GaAs the component of the dynamics characterized by the amplitude $B$ is a population-induced contribution, while the components of amplitudes $A$ and $C$ are splitting-induced contributions to the photo-induced magneto-optical Kerr effect. More precisely, the component with amplitude $A$, present only during 
the temporal coincidence of pump and probe pulses, is interpreted as a manifestation of a coherent phenomenon such as the optical Stark effect, responsible here for the lifting of the spin sub-level degeneracy.

From the fitting procedure, the component with amplitude $B$ was found to show a characteristic fast relaxation time in the order of $30 \mathrm{fs}$. Such a decay of the population-induced contribution can be due to the redistribution of carriers within the conduction band or to the ionization of excitons, the latter being highly probable at room temperature. ${ }^{26}$ The optical pump fluence used in the experiment was large enough to cause high-excitation densities. Therefore, the energy redistribution of the photo-excited electrons in the conduction band is due to both carrier-carrier and carrier-LO phonon interactions. The decay time deduced from the fit of the population-induced contribution, around $30 \mathrm{fs}$, is less than previously reported values of electron energy relaxation time (90 fs). ${ }^{22}$ This can partly be attributed to the fact that the aforementioned value was obtained at a lower temperature $(20 \mathrm{~K})$. Moreover carrier relaxation times are extremely sensitive to the excitation density and this discrepancy is also compatible with the experimental uncertainty in the determination of the pump fluence.

It should be mentioned, though, that a dependence of the relaxation time on the excitation density implies that the photo-induced magneto-optical Kerr effect is a nonlinear function of the pump fluence. A population-induced contribution is expected to have such a nonlinear dependence on the pump fluence, in contrast to a splitting-induced contribution that is linearly proportional to the pump fluence. However, as mentioned above, resolving the different contributions at short delay times $\tau_{d}$ is difficult, given the fact that our pulse width is about $100 \mathrm{fs}$, and we found no clear evidence of a nonlinear dependence of the population-induced contribution.
The splitting-induced contribution of amplitude $C$ shows a decay time between 5 and $10 \mathrm{ps}$ for intrinsic GaAs, and between 15 and 35 ps for $n$-doped GaAs. In the frame of the theoretical analysis presented above, this decay time can be assumed to be due to electron-spin relaxation. Our experimental values are in reasonable agreement with spin relaxation times obtained in other time-resolved experiments. ${ }^{27,28}$

\section{CONCLUSIONS}

Time-resolved measurements of the photo-induced, nearresonant magneto-optical Kerr effect between 1.44 and 1.63 $\mathrm{eV}$ were performed with an all-optical pump and probe technique in $n$-doped and intrinsic GaAs at room temperature. Three components to the Kerr effect were found. A theoretical analysis of these contributions was conducted. Two of them showed a similar spectral dependence and were attributed to the spin-dependent splitting of the energy levels. The first one, the duration of which does not exceed the crosscorrelation width of the pump and probe pulses, was explained in terms of a coherent effect such as the optical Stark effect. The longer decay time of the second one, up to $35 \mathrm{ps,}$ was attributed to electron-spin relaxation. The third component, exhibiting a different spectral behavior, was assumed to be due to the difference in population of the photo-excited states. Its fast decay, within the pulse duration, was attributed to the energy relaxation of the hot electrons towards the bottom of the conduction band through carrier-carrier and carrier-LO phonon scattering processes.

\section{ACKNOWLEDGMENTS}

The authors are grateful to A. V. Petukhov, B. Koopmans, and I. A. Nazarov for fruitful discussions. This work was supported in part by NWO, INTAS, RFBR, and the EU TMR Network NOMOKE.
${ }^{1}$ J. M. Kikkawa and D. D. Awschalom, Nature (London) 397, 139 (1999); I. Malajovich, J. M. Kikkawa, D. D. Awschalom, J. J. Berry, and N. Samarth, Phys. Rev. Lett. 84, 1015 (2000).

${ }^{2}$ S. Bar-Ad and I. Bar-Joseph, Phys. Rev. Lett. 68, 349 (1992).

${ }^{3}$ C. Buss, R. Pankoke, P. Leisching, J. Cibert, R. Frey, and C. Flytzanis, Phys. Rev. Lett. 78, 4123 (1997).

${ }^{4}$ J. Güdde, U. Conrad, V. Jähnke, J. Hohlfeld, and E. Matthias, Phys. Rev. B 59, R6608 (1999).

${ }^{5}$ T. Elsaesser, J. Shah, L. Rota, and P. Lugli, Phys. Rev. Lett. 66, 1757 (1991).

${ }^{6}$ B. Koopmans, M. van Kampen, J. T. Kohlhepp, and W. J. M. de Jonge, Phys. Rev. Lett. 85, 844 (2000).

${ }^{7}$ D. T. Pierce and F. Meier, Phys. Rev. B 13, 5484 (1976).

${ }^{8}$ M. I. Dyakonov and V. I. Perel, in Optical Orientation, edited by F. Meyer and B. P. Zakharchenya (North-Holland, Amsterdam, 1984).

${ }^{9}$ A. V. Kimel, V. V. Pavlov, R. V. Pisarev, V. N. Gridnev, F. Bentivegna, and Th. Rasing, Phys. Rev. B 62, R10 610 (2000).

${ }^{10}$ A. Leitenstorfer, A. Lohner, K. Rick, P. Leisching, T. Elsaesser, T. Kuhn, F. Rossi, W. Stolz, and K. Ploog, Phys. Rev. B 49, 16 372 (1994).
${ }^{11}$ S. C. Jain and D. J. Roulston, Solid-State Electron. 34, 453 (1991).

${ }^{12}$ B. Koopmans, J. E. M. Haverkort, W. J. M. de Jong, and G. Karczevski, J. Appl. Phys. 85, 6763 (1999).

${ }^{13}$ Y. R. Shen, The Principles of Nonlinear Optics (Wiley, New York, 1984).

${ }^{14}$ A. K. Zvezdin and V. A. Kotov, Modern Magnetooptics and Magnetooptical Materials (Institute of Physics Publishing, Bristol, 1997).

${ }^{15}$ L. D. Landau and E. M. Lifshitz, Electrodynamics of Continuous Media (Pergamon Press, Oxford, 1984).

${ }^{16}$ J. Frey, R. Frey, and C. Flytzanis, Phys. Rev. B 45, 4056 (1992).

${ }^{17} \mathrm{H}$. Haug and S. W. Koch, Quantum Theory of the Optical and Electronic Properties of Semiconductors (World Scientific, Singapore, 1993).

${ }^{18}$ L. Viña, L. Muñoz, E. Pẽrez, J. Fernández-Rossier, C. Tejedor, and K. Ploog, Phys. Rev. B 54, R8317 (1996).

${ }^{19}$ Th. Östreich, K. Schönhammer, and L. J. Sham, Phys. Rev. Lett. 75, 2554 (1995); L. J. Sham, J. Magn. Magn. Mater. 200, 219 (1999). 
${ }^{20}$ J. Shah, Ultrafast Spectroscopy of Semiconductors and Semiconductor Nanostructures (Springer, Berlin, 1996).

${ }^{21}$ J. A. Kenrow, K. El Sayed, and C. J. Stanton, Phys. Rev. B 58, R13 399 (1998).

${ }^{22}$ M. Betz, G. Göger, A. Leitenstorfer, K. Ortner, C. R. Becker, G. Böhm, and A. Laubereau, Phys. Rev. B 60, R11 265 (1999).

${ }^{23}$ K. Hannewald, S. Glutsch, and F. Bechstedt, Phys. Rev. B 61, 10 792 (2000).

${ }^{24}$ N. Linder and L.J. Sham, Physica E (Amsterdam) 2, 412 (1998).

${ }^{25}$ P. C. Becker, H. L. Fragnito, C. H. Brito Cruz, R. L. Fork, J. E.
Cunningham, J. E. Henry, and C. V. Shank, Phys. Rev. Lett. 61, 1647 (1988).

${ }^{26}$ W. H. Knox, R. L. Fork, M. C. Downer, D. A. B. Miller, D. S. Chemla, C. V. Shank, A. C. Gossard, and W. Wiegmann, Phys. Rev. Lett. 54, 1306 (1985).

${ }^{27}$ J. M. Kikkawa and D. D. Awschalom, Phys. Rev. Lett. 80, 4313 (1998).

${ }^{28}$ A. R. Bungay, S. V. Popov, I. R. Shatwell, and N. I. Zheludev, Phys. Lett. A 234, 379 (1997). 